\title{
HECKE INVARIANTS OF KNOT GROUPS
}

\author{
by ROBERT RILEY
}

(Received 9 September, 1972)

For each characteristic $p$, let $F_{p}$ be the prime field and let $\Omega_{p}$ be a fixed universal field which is algebraically closed and of infinite transcendence degree over $F_{p}$. When $p=0$ we take $\Omega_{p}=\mathbb{C}$. Let $F$ be a subfield of $\Omega_{p}$ and let $R$ be an integral domain whose quotient field is $F$. We abbreviate $\operatorname{SL}(2, R), \operatorname{PGL}(2, R), \operatorname{PSL}(2, R)$ to $\operatorname{SL}(R), \operatorname{PGL}(R), \operatorname{PSL}(R)$ respectively, and we consider PSL $(R)$ as a group of projective transformations of the projective line $\mathscr{P}\left(\Omega_{p}\right)$ and of the " subline" $\mathscr{P}(F) \subset \mathscr{P}\left(\Omega_{p}\right)$. The elements of $\operatorname{PSL}(R)$ are classified by the number of fixed points they have on $\mathscr{P}(F)$. If $x \in \operatorname{PSL}(R)$ has one such fixed point $P$, then $P$ is the unique fixed point of $x$ on $\mathscr{P}\left(\Omega_{p}\right)$ and $x$ is called parabolic. All other $x$ (except the identity $E$ ) have two distinct fixed points on $\mathscr{P}\left(\Omega_{p}\right)$ and $x$ is called hyperbolic if these are on $\mathscr{P}(F)$, and elliptic otherwise. We put symbols for operators on the right.

Let $K$ be a knot type with group $\pi K$, and consider homomorphisms $\theta: \pi K \rightarrow \operatorname{PSL}\left(\Omega_{p}\right)$. We shall call such a $\theta$ a parabolic representation if $\pi K \theta$ is nonabelian and $x \theta$ is parabolic for every meridian $x \in \pi K$. The abbreviation of "parabolic representation" will henceforth be " p-rep", instead of " rep" as used in [6], [7]. Note that, because $\pi K$ is finitely generated, $\pi K \theta \subset \operatorname{PSL}(R)$ for some integral domain $R$ which is finitely generated over $F_{p}$. We call two p-reps $\theta_{1}, \theta_{2}: \pi K \rightarrow \operatorname{PSL}\left(\Omega_{p}\right)$ simply equivalent when there exists $Y \in \operatorname{PGL}\left(\Omega_{p}\right)$ such that $x \theta_{2}=Y^{-1} x \theta_{1} Y$ for all $x \in \pi K$. Suppose that an over presentation of $\pi K$ has been selected. Then, according to [7, 2$]$, there is an algebraic set $M_{0}$ whose points are in one-one correspondence with the simple equivalence classes of p-reps in PSL(C), and $M_{0}$ is defined by polynomial equations with rational integral coefficients. (There is an error in $[7, \S 2]$, but it has no influence on the present paper.) If $\theta: \pi K \rightarrow \operatorname{PSL}(\mathbb{C})$ is a p-rep we write $Z=Z(\theta)$ for the corresponding point of $M_{0}$. Suppose that $Z(\theta)$ is a generic point of a subvariety $N$ of $M_{0}$ which is also defined over $\mathbb{Z}$. Then, for each positive prime $p$ we consider $N_{p}$, the reduction of $N$ modulo $p$, which is an algebraic set in characteristic $p$ defined by reducing the polynomial equations defining $N$ modulo $p$. We shall say that the p-rep $\bar{\theta}: \pi K \rightarrow \operatorname{PSL}\left(\Omega_{p}\right)$ is a reduction of $\bar{\theta}$ modulo $p$ if $\bar{\theta}$ corresponds to a generic point $\bar{Z}$ of a component of $N_{p}$. The number of equivalence classes of reductions of $\theta$ modulo $p$ is the number, $l$ say, of components of $N_{p}$.

Suppose that $\bar{\theta}$ is a reduction of $\theta$ modulo $p$ and $\pi K \bar{\theta}$ is a transitive group of projective transformations of the projective line $\mathscr{P}\left(F_{q}\right)$, where $q$ is a power of $p$ and $F_{q}$ is the field with $q$ elements. (According to Dickson's Theorem $\mathrm{C}$ of $[7, \S 6]$, this implies that $\pi K \bar{\theta}=L_{q}$ : $=\operatorname{PSL}\left(F_{q}\right)$ except possibly when $q=9$ or $q=2^{r}$ with $r>1$.) Then $\bar{\theta}$ is a permutation representation of $\pi K$ of degree $q+1$, and there is a standard method of associating unbranched and branched $q+1$ sheeted covering spaces of $S^{3}-k$ with $\bar{\theta}$ (cf. $[1, \S 8]$ ). As in [6], we write $\mathscr{U}_{q+1}=\mathscr{U}_{q+1}(K, \bar{\theta})$ for the unbranched covering space, and $\mathscr{M}_{q+1}$ for the completion of $\mathscr{U}_{q+1}$ to a branched covering space. We call the groups $H_{1} \mathscr{U}_{q+1}:=H_{1}\left(\mathscr{U}_{q+1}, \mathbb{Z}\right), H_{1} \mathscr{M}_{q+1}:=H_{1}\left(\mathscr{M}_{q+1}, \mathbb{Z}\right)$ respectively unbranched and branched homology invariants of the pair $(K, \theta)$ at $p$. There are $l$ pairs of such invariants, and the collection of all the unbranched or branched homology invariants of $(K, \theta)$ 
at $p$ may be called respectively an unbranched or a branched parabolic invariant of $K$ at $p$. This is in accord with a suggestion of Fox in the introduction to [2]. The importance of the parabolic invariants is due to the ease of computing them and their effectiveness in distinguishing isomorphism classes of knot groups.

The image of a p-rep $\theta$ in characteristic zero is only rarely a Fuchsian group, because $\pi K \theta$ usually does not lie in $\operatorname{PSL}(\mathbb{R})$, and when $\pi K \theta \subset \mathrm{PSL}(\mathbb{R})$ we generally find that $\pi K \theta$ is indiscrete. In fact, the only Fuchsian groups $G$ known to be the image of a parabolic representation are isomorphic to the free product $\mathrm{C}_{r} * C_{s}$ of cyclic groups of relatively prime orders $r, s>1$. The modular group $\operatorname{PSL}(\mathbb{Z})$ is the simplest group of this sort and $\operatorname{PSL}(\mathbb{Z}) \approx C_{2} * C_{3}$. For each odd integer $s>1$ there is a Hecke group $\Pi_{s} \approx C_{2} * C_{s}$ (whose definition is recalled in $\S 1$ ) and the simplest Hecke group is $\Pi_{3}=$ PSL $(\mathbb{Z})$. The Hecke groups may be regarded as the immediate generalization of the modular group, and $\Pi_{s}$ is of the form $\pi K \theta$ for infinitely many knot types $K$, including 2-bridge knot types. The collection of all (unbranched or branched) homology invariants of $K$ at a prime $p$ corresponding to p-reps $\theta: \pi K \rightarrow \Pi_{s}$ may be called the (unbranched or branched) Hecke invariant of $(K, s)$ at $p$. In this paper we shall apply the highly developed theory of Fuchsian groups to establish properties of Hecke invariants which are apparently not shared by more general parabolic invariants. Our main result is Theorem 3 of $\$ 3$, which gives a lower bound for the Betti numbers of the unbranched Hecke invariants of $(K, s)$ at a prime $p$ not dividing $s$. The results of this paper are a necessary preliminary to any assault on Conjecture $B$ of [6], and our main purpose in writing this paper is to raise interest in that conjecture in the hope that someone will try to prove it.

This paper is arranged as follows. In $\S 1$ we define the Hecke groups in the precise form in which we need them, and establish their most important properties. This material is presumably well known, but, because this paper is intended for knotters rather than for experts on Fuchsian groups, we felt it wise to be explicit. In $\$ 2$ we discuss certain subgroups $\Gamma$ of $\Pi_{s}$. The material here is a generalization of classical theorems about the modular group (see page 304 of Fricke's textbook [3]) and it may also be well known, but I cannot find a reference for it. In $\$ 3$ we prove the advertised main theorem about $H_{1} \mathscr{U}_{q+1}$. Finally, in $\S 4$ we restate Conjecture $B$ in an improved form and discuss the Hecke invariants at primes $p>3$ for p-reps on $\operatorname{PSL}(\mathbb{Z})$ when $K$ is one of the three torus knots $3_{1}, 9_{1}, 8_{1}$.

This paper could not have been written without the assistance of David Singerman, who brought his paper [8] to my attention and explained how to use the results therein to prove our Theorem 2 of $\$ 2$ in the case where $\Pi_{s}$ is PSL(ZZ). A part of the proof of Theorem 3 was developed in a discussion with Gareth Jones, who is a ready source of wisdom about group theory.

1. Hecke groups. Let $s>1$ be a fixed odd integer. We define the Hecke group $\Pi_{s}$ in terms of the Morgan-Voyce polynomials $f_{j}=f_{j}(y), g_{j}=g_{j}(y)$ following $[7, \S 5]$ as follows. The Morgan-Voyce polynomials are defined inductively by

$$
\left.\begin{array}{rlrl}
f_{0} & =1, & g_{0} & =1, \\
f_{j+1} & =f_{j}+y g_{j}, & g_{j+1} & =f_{j+1}+g_{j}
\end{array}\right\}
$$

Let $s_{1}>1$ be a divisor of $s$ and let $n_{1}=\frac{1}{2}\left(s_{1}-1\right)$. In particular, let $n=\frac{1}{2}(s-1)$. Then there is a factor $\chi_{n_{1}}(y)$ of $f_{n}(y)$ and 


$$
f_{n}(y)=\prod_{s_{1} / s} \chi_{n_{1}}(y) .
$$

The degree of $\chi_{n_{1}}(y)$ is $\frac{1}{2} \phi\left(s_{1}\right)$, where $\phi$ is the Euler function. We are mainly interested in $\chi_{n}(-y)$ whose roots are

where

$$
\xi_{j}=2+\dot{2} \cos 2 \pi j / s=(2 \cos \pi j / s)^{2},
$$

$$
1 \leqq j<s, \quad(j, s)=1 .
$$

Note that $\xi_{j}=\xi_{s-j}$. The largest root of $\chi_{n}(-y)=0$ (which is also the largest root of $\left.f_{n}(-y)=0\right)$ is $\xi=\xi_{1}=\xi_{s-1}$. Let

$$
A=\left(\begin{array}{ll}
1 & 1 \\
0 & 1
\end{array}\right), \quad B=B(\xi)=\left(\begin{array}{cc}
1 & 0 \\
-\xi & 1
\end{array}\right) \quad \text { in } \operatorname{PSL}(\mathbb{R}) .
$$

Then we define the Hecke group $\Pi_{s}$ by

$$
\Pi_{s}=\langle A, B\rangle .
$$

THEOREM 1. The Hecke group $\Pi_{s}$ is a Fuchsian group with a presentation

which is related to (1.5) by

$$
\Pi_{s}=\left|C, D: C^{2}=D^{s}=E\right|
$$

$$
C=(A B)^{n} A, \quad D=A B, \quad A=D^{-n} C, \quad B=A^{-1} D .
$$

Proof. The only part of the proposition which is not contained in Theorem 6 of [7] is the assertion that $\Pi_{s}$ is Fuchsian, i.e., that $\Pi_{s}$ acts discontinuously on the upper half-plane. To verify this, we need the explicit formulae

$$
\begin{aligned}
& D=\left(\begin{array}{cc}
1-\xi & 1 \\
-\xi & 1
\end{array}\right), \\
& C=\left(\begin{array}{cc}
0 & g_{n-1}(-\xi) \\
-\xi g_{n-1}(-\xi) & 0
\end{array}\right) .
\end{aligned}
$$

The elements $C, D \in \operatorname{PSL}(\mathbb{R})$ are elliptic linear fractional transformations of the upper halfplane $\mathscr{U}=\{z \in \mathbb{C} \mid \operatorname{Im}(z)>0\}$. Hence each transformation has a unique fixed point in $\mathscr{U}$, say $P_{0} D=P_{0}$ and $Q_{0} C=Q_{0}$. We calculate readily that

$$
P_{0}=\frac{1}{2}+\frac{1}{2} \sqrt{ }(1-4 / \xi), \quad Q_{0}=\frac{1}{\sqrt{ }(-\xi)} .
$$

Another calculation shows that $P_{0}$ and $Q_{0}$ have the same euclidean distance from the point $O=(0,0)$ on the euclidean boundary $V$ of $\mathscr{U}$. In terms of the standard non-euclidean (NE) metric on $\mathscr{U}$ in which the geodesics are euclidean semicircles with centres on $V$ or euclidean straight lines perpendicular to $V$, let $s_{1}$ be the NE line segment $P_{0} Q_{0}$. Let $s_{0}$ be the NE segment $P_{0} \infty=\left\{\frac{1}{2}+\mathrm{i} t \mid t \geqq \frac{1}{2} \sqrt{ }(4 / \xi-1)\right\}$, and let $s_{2}=P_{0} Q_{1}$ be the reflection of $s_{1}$ in $s_{0}$. Let $s_{3}=\{$ it $\mid t \geqq 1 / \sqrt{ } \xi\}$ be the NE segment $Q_{0} \infty$, and let $s_{4}$ be the refiection of $s_{3}$ in $s_{0}$. Then $s_{4}=\{1+\mathrm{i} t \mid t \geqq 1 / \sqrt{ } \xi\}$ has finite endpoint $Q_{1}, Q_{1}=Q_{0} A$ and $s_{4}=s_{3} A$. If we close the 
segments $s_{3}, s_{4}$ by the point $\infty$, the path $s_{1} s_{2} s_{4} s_{3}$ is the boundary of an open NE polygonal region $R$ of $\mathscr{U}$. It is easy to see that $s_{1}$ meets $s_{3}$ in a right angle at $Q_{0}$, and likewise $s_{2}$ meets $s_{4}$ in a right angle at $Q_{1}$. The angle at $P_{0}$ between $s_{1}$ and $s_{2}$ is bisected by $s_{0}$; let $\theta=$ angle $s_{1} s_{0}=$ angle $s_{0} s_{2}$. Let $P_{*}=\left(\frac{1}{2}, 0\right) \in V$ and consider the euclidean right triangle $P_{0} O P_{*}$. The angle $P_{0} O P_{*}=\theta$ and $\cos \theta=O P_{*} / O P_{0}=\frac{1}{2} /(2 \cos \pi / s)^{-1}=\cos \pi / s$. But $0<\theta<\pi / 2$; so $\theta=\pi / s$. The transformation $D$ is a NE rotation of exact period $s$ about the fixed point $P_{0}$; so the angle of rotation necessarily has the form $2 \pi j / s$ for some integer $j$ such that $(j, s)=1$.

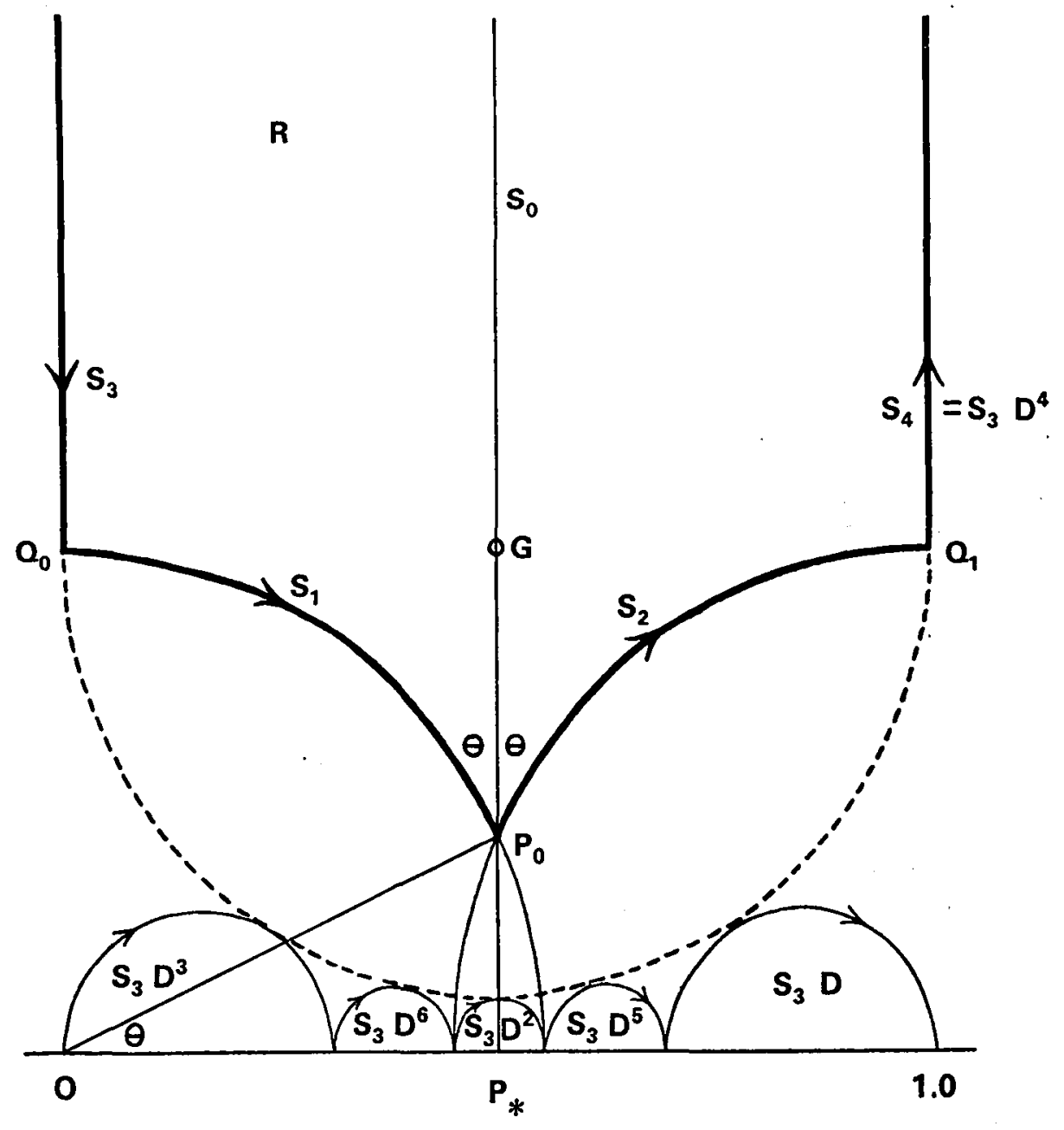

The situation when $s=7, \xi=3.2470$.

$\mathrm{G}$ is the centre of the dashed circle. The transforms of $s_{3}$ by powers of $D$ are indicated by $s_{3}, s_{3} D, \ldots, s_{3} D^{6}$. 
(The value of $j$ is irrelevant but it turns out that $j=-2$.) Consequently, for some exponent $r$, $s_{2}=s_{1} D^{r}$. Finally note that $\langle A, D\rangle=\Pi_{s}$, from (1.7).

We now appeal to the theorem of Poincaré stated in Note 6, page 64 of Chapter 1 of [4] and proved in [5]. The conclusion is that $\Pi_{s}$ acts discontinuously on $\mathscr{U}$ and that $R$ is a fundamental open region for the action. Poincaré's theorem also verifies the completeness of the relations in (1.6), and this method should be taken as the standard proof of Theorem 1.

The final reference, [9], appeared several months after the present paper was submitted for publication. It is closely related to the contents of this section, and it contains a reference to E. Hecke's own work on Hecke groups.

2. Subgroups of Hecke groups. This section is devoted to the application of the material summarized in the first two sections of Singerman's paper [8] to the determination of the genus of a certain subgroup $\Gamma$ of a Hecke group $\Pi_{s}$. It is convenient to define the class of the element $g$ of a group $G$ to be the set of all conjugates of powers $g^{\nu}$ of $g$ such that $g^{\nu} \neq E$ if $g \neq E$. By $\mathfrak{U} G$ we mean $G /[G, G]$.

A Fuchsian group $G$ which has no hyperbolic boundary elements has a canonical presentation as follows:

$$
\begin{aligned}
& \text { Generators: }\left\{\begin{array}{ll}
a_{1}, b_{1}, \ldots, a_{g}, b_{g} & \text { hyperbolic, } g=\operatorname{genus}(G), \\
u_{1}, u_{2}, \ldots, u_{h} & \text { elliptic, } \\
v_{1}, v_{2}, \ldots, v_{t} & \text { parabolic. }
\end{array}\right\} \\
& \text { Relations: } u_{1}^{m_{1}}=u_{2}^{m_{2}}=\ldots=u_{h}^{m_{h}}=\prod_{i=1}^{g}\left[a_{i}, b_{i}\right] \cdot \prod_{j=1}^{h} u_{i} \cdot \prod_{k=1}^{t} v_{k}=E .
\end{aligned}
$$

Every nontrivial element of $G$ is in the class of exactly one of the generators (2.1a). When $G$ is presented by $(2.1)$, define $M(G)$ by

$$
M(G)=2 g-2+t+\sum_{i=1}^{h}\left\{1-\frac{1}{m_{i}}\right\} .
$$

The Riemann-Hurwitz formula asserts that, if $G_{1} \subset G$ is a subgroup of finite index $N=\left[G: G_{1}\right]$, then

$$
N=M\left(G_{1}\right) / M(G)
$$

The presentation (1.6) for $\Pi_{s}$ is not quite in the form (2.1), but this is no problem because it is readily apparent from (1.6) that the genus of $\Pi_{s}$ is zero, that the number of parabolic classes $t=1$ (certainly $t \geqq 1$, and $t>1$ would give $\mathfrak{A} \Pi_{s}$ a torsion-free direct summand), that $h=2$ and $m_{1}=2, m_{2}=s$, say. Consequently

$$
M\left(\Pi_{s}\right)=\frac{s-2}{2 s} .
$$

Let $p$ be an odd prime which does not divide $s$. Then the factorization of $\chi_{n}(y)$ modulo $p$ has the form

$$
\chi_{n}(y) \equiv \chi^{(1)}(y) \ldots \chi^{(l)}(y) \quad(\bmod p)
$$


where

$$
\operatorname{deg} \chi^{(1)}=\operatorname{deg} \chi^{(2)}=\ldots=\operatorname{deg} \chi^{(l)}=\frac{\phi(n)}{2 l}=r, \text { say. }
$$

For each $j$ with $1 \leqq j \leqq l$, let $\xi_{j} \in \Omega_{p}$ be a root of $\chi^{(j)}$. Then there is a surjective homomorphism $\phi_{p}^{j^{\prime}}: \Pi_{s} \rightarrow L_{p^{r}}$ defined by

$$
A \phi_{p}^{j}=\left(\begin{array}{ll} 
& 1 \\
0 & 1
\end{array}\right), \quad B \phi_{p}^{j}=\left(\begin{array}{cc}
1 & 0 \\
-\xi_{j} & 1
\end{array}\right)
$$

in $\operatorname{PSL}\left(\Omega_{p}\right)$. Then $\phi_{p}^{j}$ is called a reduction of $\Pi_{s}$ modulo $p$. Up to equivalence there are exactly $l$ such reductions, where two homomorphisms $\theta_{1}, \theta_{2}: G_{1} \rightarrow G_{2}$ are equivalent if $G_{2}$ has an automorphism $\psi$ such that $\theta_{2}=\theta_{1} \psi$. Let $\Pi_{s, j}^{\infty}(p)$ denote the subgroup of $\Pi_{s}$ consisting of all $\gamma$ such that the projective transformation $\gamma \phi_{p}^{j}$ acting on the projective line $\mathscr{P}\left(F_{p r}\right)$ leaves $\infty$ fixed. To simplify the notation we fix one reduction $\phi_{p}^{j}$ and write

$$
\phi=\phi_{p}^{j}, \quad \Gamma=\Pi_{s, j}^{\infty}(p), \quad \xi=\xi_{j} .
$$

We also set $q=p^{r}$, where $r$ is defined in (2.6).

The main result of this section is a presentation for the abelianized group $\mathfrak{A} \Gamma=\Gamma /[\Gamma, \Gamma]$. We will get this by computing the various quantities $h, m_{1}, \ldots, m_{h}, t, g$ of a canonical presentation (2.1) for $\Gamma$. The parabolic class number $t$ is, according to the summary in [8], the number of cycles in $A \phi$, considered as a permutation of the points of $\mathscr{P}\left(F_{q}\right)$. But $A \phi$ has one fixed point and order $p$, whence $A \phi$ must be the product of $q / p p$-cycles and one 1-cycle. This implies that

$$
t=p^{r-1}+1 \text {. }
$$

We next consider $h$ and the $m_{i}$. The $u_{i}$ of (2.1) for $\Gamma$ are induced (in Singerman's language) by the elliptic elements $C, D$ of (1.7). The involution $C$ induces $v$ elliptic elements $u$ of $\Gamma$, where $v$ is the number of cycles of the permutation $C \phi$ of length less than 2. Such cycles must be fixed points; so either $C$ induces no elliptic elements, or else $C$ induces two elliptic elements and these are involutions. From (1.9) and (1.10) we see that the number of classes of involutions induced by $C$ is the number of solutions of

$$
z^{2}+\bar{a}^{2}=0, \quad \bar{a}=g_{n-1}(-\bar{\xi})
$$

in $F_{q}$. Clearly (2.10) has solutions in $F_{q}$ if and only if -1 is a square in $F_{q}$, or, equivalently, if and only if $q \equiv+1(\bmod 4)$.

Now consider the elliptic elements $u$ of $\Gamma$ induced by $D$. Since $D$ has order $s, D \phi$ has order $s_{1}$, where $s_{1} \mid s$. To see that $s_{1}=s$, consider the multiplier $\kappa$ of $D$ and the multiplier $\bar{\kappa}$ of $D \phi$. Then $\kappa$ is a root of the cyclotomic polynomial $\Phi_{s}(x)$, and we recall that

$$
x^{s}-1=\prod_{m / s} \Phi_{m}(x) \text {. }
$$

It is well known that $x^{s}-1$ has no multiple factors modulo $p$, so that, in $\Omega_{p}[x]$,

$$
x^{s}-1=\prod_{m \mid s} \bar{\Phi}_{m}(x)
$$


where $\bar{\Phi}_{m}$ is the reduction of $\Phi_{m}(\bmod p)$. Also, $\bar{\kappa}$ is a root of $\bar{\Phi}_{s}(x)$. Hence a comparison of degrees shows that, if $\bar{\kappa}^{\nu}=1$, then $s \mid v$, which implies that $s_{1}=s$. The permutation $D \phi$ may contain only $s$-cycles and 1-cycles, and furthermore there are either two or zero 1-cycles. Hence $q+1 \equiv$ the number of fixed points of $D \phi(\bmod s)$, so that, if $q \equiv+1(\bmod s)$, then $D$ induces two elliptic elements of order $s$, and, if $q \equiv-1(\bmod s)$, then $D$ induces no elliptic elements of $\Gamma$.

We finally determine the genus of $\Gamma$ indirectly using (2.3) and the information about $M(\Gamma)$ deduced from the above results. The index $N$ of $(2.3)=q+1=$ the number of points of $\mathscr{P}\left(F_{q}\right)$. Hence

$$
q+1=\frac{2 g-2+1+p^{r-1}+\Sigma}{[(s-2) / 2 s]}, \quad \Sigma:=\sum_{i=1}^{h}\left\{1-\frac{1}{m_{i}}\right\} .
$$

The calculation splits into four cases and the results are summarized in the following theorem.

THEOREM 2. The abelianized group $\mathfrak{M} \Gamma$ depends only on $s$ and $p$, and not on the specific reduction $\phi_{p}^{j}$ of $\Pi_{s}$. Write $\mathfrak{U} \Gamma=F \oplus T$ where $T$ is the torsion subgroup and $F$ is a free abelian summand of rank $f_{s}(p)$. Then the genus $g=g_{s}(p)$ of $\Gamma, f=f_{s}(p)$ and the torsion numbers of $T$ are determined by the value of $q(\bmod 4)$ and $q(\bmod s)$, by

and the following table.

$$
f_{s}(p)=2 g_{s}(p)+p^{r-1}
$$

$$
\begin{aligned}
& q \equiv+1(\bmod 4) \text { and } q \equiv+1(\bmod s) \\
& g=(s-2) \frac{p^{r}-1}{4 s}-\frac{p^{r-1}+1}{2} ; \text { torsion numbers } 2,2, s, s . \\
& q \equiv+1(\bmod 4) \text { and } q \equiv-1(\bmod s) . \\
& g=\frac{p^{r}-2 p^{r-1}+1}{4}-\frac{p^{r}+1}{2 s} ; \text { torsion numbers } 2,2 . \\
& q \equiv-1(\bmod 4) \text { and } q \equiv+1(\bmod s) . \\
& g=\frac{p^{r}-2 p^{r-1}-1}{4}-\frac{p^{r}-1}{2 s} ; \text { torsion numbers } s, s . \\
& q \equiv-1(\bmod 4) \quad \text { and } q \equiv-1(\bmod s) . \\
& g=(s-2) \frac{p^{r}+1}{4 s}-\frac{p^{r-1}-1}{2} ; \text { no torsion. }
\end{aligned}
$$

3. The homology of the unbranched covering spaces. Let $\pi K$ be a knot group which has a surjective parabolic representation $\theta: \pi K \rightarrow \Pi_{s}$ for some odd $s>1$.

LEMMA. There is a presentation

$$
\pi K=\left|x_{1}, \ldots, x_{v}: r_{2}, \ldots, r_{v}\right|
$$


in which the generators $x_{i}$ are conjugate meridians of $\pi K$ and such that $\theta$ can be normalized with respect to (3.1) so that

where $A, B$ are defined in (1.4).

$$
x_{1} \theta=A, \quad x_{2} \theta=B
$$

Proof. We start with an arbitrary over-presentation

$$
\pi K=\left|\tilde{x}_{1}, \ldots, \tilde{x}_{v-1}: \tilde{r}_{2}, \ldots, \tilde{r}_{v-1}\right| .
$$

The representation $\theta$ can be normalized with respect to (3.3) so that $\tilde{x}_{1} \theta=A$. Since $B$ is conjugate to $A$ in $\Pi_{s}$, there is a $W \in \Pi_{s}$ such that $B=W^{-1} A W$. Because $\theta$ is surjective there is a $w \in \pi K$ such that $W=w \theta$. Let $x=w^{-1} \tilde{x}_{1} w$; then $x$ is a meridian of $\pi K$ which is conjugate to $x_{1}$ and satisfies $x \theta=B$. It is now obvious that, by adjoining the new generator $x$ and the relation $x=w^{-1} \tilde{x}_{1} w$ to (3.3) (with $w$ written out as a word in $\tilde{x}_{1}, \ldots, \tilde{x}_{v-1}$ ), and suitably renaming the generators and relations, we produce the required presentation.

Let $p$ be an odd prime not dividing $s$ and let $\phi=\phi_{p}^{j}$ be one of the $l$ reductions of $\Pi_{s}$ modulo $p$. Then $\phi: \Pi_{s} \rightarrow L_{q}$, where $q=p^{r}$ and $r$ is determined by (2.6). Again write $\Gamma=\Pi_{s, j}^{\infty}(p)$ and let $M=\theta^{-1}(\Gamma)$ be the pre-image of $\Gamma$ in $\pi K$. Then the unbranched $(q+1)-$ sheeted covering space $\mathscr{U}_{q+1}$ of the introduction has fundamental group $\pi_{1} \mathscr{U}_{q+1} \approx M$. Consequently $H_{1} \mathscr{U}_{q+1} \approx \mathfrak{M} M$ and we are ready to apply Theorem 2 .

THeOREM 3. The Betti number $b=b(K, \theta, j, p)$ of $H_{1} \mathscr{U}_{q+1}$ satisfies

$$
b \geqq 1+f_{s}(p) \text {, }
$$

where $f_{s}(p)$ is defined in Theorem 2.

Proof. Write $H=\operatorname{ker} \theta$. The system of groups and homomorphisms under consideration is summarized by the commutative diagram (3.5) in which the rows are exact, the vertical arrows represent canonical inclusion homomorphisms, and $\theta^{\prime}, \theta^{\prime \prime}$ denote restrictions of $\theta$ to the appropriate subgroups.

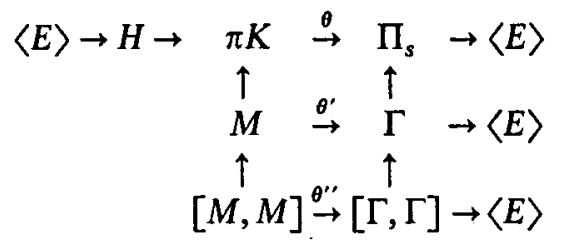

It is easy to see that $\theta$ induces a surjective homomorphism $\theta_{\boldsymbol{*}}: \mathfrak{U} M \rightarrow \mathfrak{U} \Gamma$. This shows that $b \geqq f$. To show that equality does not hold it is sufficient to exhibit $h \in H$ such that no nonzero power of $h$ lies in $[M, M]$, because $h$ would then generate a free summand of $\operatorname{ker} \theta_{*}$. Suppose that $\theta$ has been normalized as in the lemma. Let $w_{1}=\left(x_{1} x_{2}\right)^{n} x_{1} \in \pi K$, where $n=\frac{1}{2}(s-1)$ as usual. Then $w_{1} \theta$ is the element $C$ of $(1.7)$; so $h_{1}:=w_{1}^{2}$ must lie in $\operatorname{ker} \theta=H$. But $h_{1}$ represents $s+1 \neq 0$ in $\mathfrak{I} \pi K \approx \mathbb{Z}$, whence $h_{1}^{m} \notin[\pi K, \pi K]$ for any nonzero $m$.

4. Conjecture B. In this section we consider the relation between the two homology invariants $H_{1} \mathscr{U}_{p+1}, H_{1} \mathscr{M}_{p+1}$ at a prime $p>3$ of a p-rep $\theta: \pi K \rightarrow \Pi_{3}=\operatorname{PSL}(\mathbb{Z})$. In this case 
the polynomial $\chi_{n}(y)$ is $1+y$ and the numbers $r, l$ of (2.6) are both 1 . The formulae of Theorem 2 simplify to $f_{3}(p)=1+2 g(p)$, where

$$
g(p)=\frac{p+j(p)}{12},
$$

in which $j(p)$ is determined by the value of $p(\bmod 12)$ as follows.

$$
\left.\begin{array}{rlrrrr}
p & \equiv 1 & 5 & 7 & 11 & (\bmod 12) \\
j(p) & =-13 & -5 & -7 & +1 \\
r(p) & =1 & 3 & 2 & 6
\end{array}\right\} \quad
$$

The lower bound $1+f$ of Theorem 3 becomes $2+2 g(p)$. We shall also need

$$
d(p)=\frac{p+1}{r(p)},
$$

where $r(p)$ is defined by the bottom line of (4.2). As further notation, let $b X$ denote the Betti number of $H_{1} X:=H_{1}(X ; \mathbb{Z})$ and let $T_{1} X$ denote the torsion subgroup of $H_{1} X$. Then the desired relation is the

SHARPENEd CONJECTURE B. Given $\theta: \pi K \rightarrow \operatorname{PSL}(\mathbb{Z})$ and a prime $p>3$, the relation between the homology invariants $H_{1} \mathscr{U}_{p+1}, H_{1} \mathscr{M}_{p+1}$ is as follows.

$$
b \mathscr{U}_{p+1}=2+b \mathscr{M}_{p+1} .
$$

According to Theorem 3 the assertion 4.4 implies that

$$
b \mathscr{M}_{p+1} \geqq 2 g(p) \text {. }
$$

Write $m_{p}$ for the order of $T_{1} \mathscr{M}_{p+1}$ and $u_{p}$ for the order of $T_{1} \mathscr{U}_{p+1}$. Then

$$
\begin{gathered}
b \mathscr{M}_{p+1}=2 g(p) \Rightarrow m_{p}=d(p) u_{p} ; \\
b \mathscr{M}_{p+1}>2 g(p) \Rightarrow m_{p} / u_{p} \text { is an integer which divides } d(p) .
\end{gathered}
$$

No general assertion is made about the comparison of the torsion coefficients of $T_{1} \mathscr{U}_{p+1}$, $T_{1} \mathscr{H}_{p+1}$.

The final topic is the question whether the homology invariants for a p-rep $\theta: \pi K \rightarrow \operatorname{PSL}(\mathbb{Z})$ can be computed as functions of $p$ by simple formulae. It seems that they can be, at least when $K$ is a torus knot. A general answer to this question would be both important and interesting, and might be a step towards a substitute for the Alexander matrix which solves the corresponding problem for cyclic representations. To encourage further investigations along these lines we present conjectured formulae for the cases $K=3_{1}, 9_{1}$ and $8_{19}$. Our tables suggest that formulae probably exist for non-torus knots such as $8_{5}, 8_{18}$ which are similar to, but more complicated than, those given below. Unfortunately, much more data would be required to guess them, and the machine time required to calculate the invariants at $p$ is a rapidly increasing function of $p$.

We use the notation of $[6, \S 6]$ to describe groups $T_{1} X$; i.e., if $T_{1} X$ is the direct sum of 
cyclic groups of orders $a_{1}, \ldots, a_{m}$, we write $T_{1} X=a_{1}, \ldots, a_{m}$. These will always be ordered so that $a_{j} \mid a_{j+1}$ for $j<m$. When the torsion number $a$ is repeated $e$ times we abbreviate $a, \ldots, a$ to $a^{(e)}$.

The knot groups $\pi K$ for $K=3_{1}, 9_{1}, 8_{19}$ each have a unique p-rep on PSL(Z) listed in [6, §3], using the table of group presentations in the microfiche section of the issue of Mathematics of Computation containing [6]. It is conjectured that the relations $b \mathscr{M}_{p+1}=2 g(p)$, $b \mathscr{U}_{p+1}=2+b \mathscr{M}_{p+1}$ hold for all primes $p>3$ for these three knots. The torsion invariants are:

$$
\begin{aligned}
& K=3_{1}\left\{\begin{array}{l}
T_{1} \mathscr{U}_{p+1}=\frac{6}{r(p)} \\
T_{1} \mathscr{M}_{p+1}=\frac{6 d(p)}{r(p)}
\end{array}\right. \\
& K=9_{1}\left\{\begin{array}{l}
T_{1} \mathscr{U}_{p+1}=3^{\left(e_{p}\right)}, \frac{18}{r(p)} \\
T_{1} \mathscr{M}_{p+1}=3^{\left(e_{p}\right)}, \frac{18 d(p)}{r(p)}
\end{array}\right\} \text { where } e_{p}=\left\{\begin{array}{l}
\frac{p-1}{3}(p \equiv 1(\bmod 3)), \\
\frac{p-5}{3}(p \equiv 2(\bmod 3)) .
\end{array}\right. \\
& K=8_{19}\left\{\begin{array}{c}
T_{1} \mathscr{U}_{p+1}=2^{\left(h_{p}\right)}, \frac{12}{r(p)} \\
T_{1} \mathscr{M}_{p+1}=2^{\left(h_{p}\right)}, \frac{12 d(p)}{r(p)}
\end{array}\right\} \text { where } h_{p}=\left\{\begin{array}{l}
\frac{p-1}{2}(p \equiv 1(\bmod 4)), \\
\frac{p-3}{2}(p \equiv 3(\bmod 4)) .
\end{array}\right.
\end{aligned}
$$

\section{REFERENCES}

1. R. H. Fox, A quick trip through knot theory, Topology of 3-manifolds and related topics (Englewood Cliffs, N.J., 1962), 120-167.

2. R. H. Fox, Metacyclic invariants of knots and lines, Canad. J. Math. 22 (1970), 193-201.

3. R. Fricke, Lehrbuch der Algebra, Dritter Band (Braunschweig, 1928).

4. J. Lehner, A short course in automorphic functions (New York, 1966).

5. B. Maskit, On Poincaré's theorem for fundamental polygons, Advances in Mathematics 7 (1971), 219-230.

6. R. Riley, Homomorphisms of knot groups on finite groups, Math. Comp. 25 (1971), 603-619.

7. R. Riley, Parabolic representations of knot groups, I, Proc. London Math. Soc. (3) 24 (1972), 217-242.

8. D. Singerman, Subgroups of Fuchsian groups and finite permutation groups, Bull. London Math. Soc. 2 (1970), 319-323. 115.

9. R. Evans, A fundamental region for Hecke's modular group, J. Number Theory 5 (1973), 108-

\section{UNIVERSITY OF SOUTHAMPTON}

